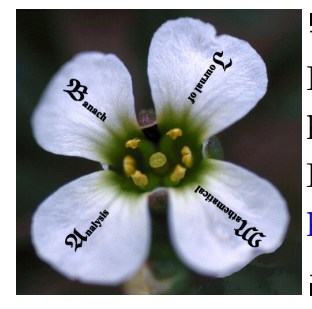

Banach J. Math. Anal. 9 (2015), no. 2, 248-252

http://doi.org/10.15352/bjma/09-2-16

ISSN: $1735-8787$ (electronic)

http://projecteuclid.org/bjma

\title{
WEAK APPROXIMATION PROPERTIES OF SUBSPACES
}

\author{
JU MYUNG KIM ${ }^{1}$ AND KEUN YOUNG LEE*2
}

Communicated by D. E. Alspach

\begin{abstract}
The paper is concerned with weak approximation properties which are weaker than the classical approximation property. For $\lambda \geq 1$, we prove that a Banach space $X$ has the $\lambda$-bounded weak approximation property $(\lambda$ BWAP) if and only if every locally 1-complemented subspace of $X$ has the $\lambda$-BWAP, and that if $X$ has the $\lambda$-BWAP and $Z$ is a locally $\mu$-complemented subspace of $X$, then $Z$ has the $(2 \mu+4) \mu \lambda$-BWAP. It also follows that $X$ has the weak approximation property (WAP) if and only if every locally complemented subspace of $X$ has the WAP.
\end{abstract}

\section{Introduction and Main Results}

A Banach space $X$ is said to have the approximation property (AP) if for every compact subset $K$ of $X$ and every $\varepsilon>0$, there exists a finite rank and continuous linear map (operator) $S$ on $X$ such that $\sup _{x \in K}\|S x-x\| \leq \varepsilon$, briefly, $i d_{X} \in$ $\overline{\mathcal{F}}(X)^{\tau_{c}}$, where $i d_{X}$ is the identity map on $X, \mathcal{F}(X)$ is the space of all finite rank operators on $X$ and $\tau_{c}$ is the topology of uniformly compact convergence on the space $\mathcal{L}(X)$ of all operators on $X$. For $\lambda \geq 1$, if $i d_{X} \in \overline{\{S \in \mathcal{F}(X):\|S\| \leq \lambda\}}{ }^{\tau_{c}}$, then we say that $X$ has the $\lambda$-bounded approximation property $(\lambda$-BAP). Choi and the first author $[1,7]$ introduced and studied weaker forms of the AP. A Banach space $X$ is said to have the weak approximation property (WAP) if $\mathcal{K}(X) \subset$ $\overline{\mathcal{F}(X)}{ }^{\tau_{c}}$, where $\mathcal{K}(X)$ is the space of all compact operators on $X$. For $\lambda \geq 1$,

Date: Received: Jul. 4, 2014; Revised: Aug. 11, 2014; Accepted: Aug. 27, 2014.

* Corresponding author.

2010 Mathematics Subject Classification. Primary 46B28; Secondary 47L20.

Key words and phrases. Approximation property, weak approximation property, bounded weak approximation property. 
we say that $X$ has the $\lambda$-bounded weak approximation property ( $\lambda$-BWAP) if for every $T \in \mathcal{K}(X)$, we have $T \in \overline{\{S \in \mathcal{F}(X):\|S\| \leq \lambda\|T\|\}}{ }^{\tau_{c}}$.

For $\mu \geq 1$, a closed subspace $Z$ of a Banach space $X$ is called locally $\mu$ complemented in $X$ if for every finite-dimensional subspace $E$ of $X$ and every $\varepsilon>0$, there exists an operator $T: E \rightarrow Z$ with $\|T\| \leq \mu+\varepsilon$ such that $T x=x$ for all $x \in E \cap Z$. It is well known that the AP and the BAP are inherited by locally complemented subspaces (cf. [3, Theorem 2.4]). The first author [7, Theorem 1.4] obtained the analogue for the WAP and the BWAP under an assumption. In this paper, we have:

Theorem 1.1. If $X$ has the $\lambda-B W A P$ and $Z$ is a locally $\mu$-complemented subspace of $X$, then $Z$ has the $(2 \mu+4) \mu \lambda-B W A P$.

For a closed subspace $Z$ of a Banach space $X$, a map $\Phi: Z^{*} \rightarrow X^{*}$ is called an extension operator if $\left(\Phi z^{*}\right)(z)=z^{*}(z)$ for every $z^{*} \in Z^{*}$ and $z \in Z$. A closed subspace $Z$ of $X$ is called an ideal if there exists an extension operator $\Phi$ from $Z^{*}$ to $X^{*}$ with $\|\Phi\|=1$. The operator $\Phi$ is called a Hahn-Banach extension operator. It is well known that $Z$ is an ideal in $X$ if and only if $Z$ is locally 1-complemented in $X$ (cf. Lemma 2.2), and that $X$ has the $\lambda$-BAP (resp. AP) if and only if every ideal in $X$ has the $\lambda$-BAP (resp. AP) (cf. [8, Proposition 4.3 and Theorem 2.2] and [3, Theorem 2.4]). In this paper, we have:

Theorem 1.2. Let $\lambda \geq 1$. The following statements are equivalent.

(a) $X$ has the $\lambda$-BWAP.

(b) Every ideal in $X$ has the $\lambda$-BWAP.

(c) If for every separable closed subspace $Y$ of $X$, there exists a separable closed subspace $Z$ of $X$ with $Y \subset Z$ such that $Z$ has the $\lambda$-BWAP.

Theorem 1.3. The following statements are equivalent.

(a) $X$ has the WAP.

(b) Every locally complemented subspace of $X$ has the WAP.

(c) Every ideal in $X$ has the WAP.

(d) If for every separable closed subspace $Y$ of $X$, there exists a separable closed subspace $Z$ of $X$ with $Y \subset Z$ such that $Z$ has the WAP.

\section{Proof of Theorem 1.1}

Lemma 2.1. [5, Lemma 3.2] If $Z$ is a locally $\mu$-complemented subspace of $X$, then for every closed subspace $Y$ of $X$ containing $Z$ with $\operatorname{dim} Y / Z<\infty$, there exists a projection $P$ from $Y$ onto $Z$ with $\|P\| \leq 2 \mu+4$.

The following lemma is a simple extension of [5, Theorem 3.4 and Theorem 3.5] (cf. [2]).

Lemma 2.2. Let $Z$ be a closed subspace of $X$ and let $\mu \geq 1$. The following statements are equivalent.

(a) For every Banach space $Y$ and every $T \in \mathcal{K}(Z, Y)$, there exists a $\widetilde{T} \in \mathcal{K}(X, Y)$ with $\|\widetilde{T}\| \leq \mu\|T\|$ such that $\widetilde{T} x=T x$ for all $x \in Z$.

(b) $Z$ is locally $\mu$-complemented in $X$. 
Proof of Theorem 1.1. Let $T \in \mathcal{K}(Z)$. Let $K$ be a compact subset of $Z$ and let $\varepsilon>0$ be given. Since $Z$ is locally $\mu$-complemented in $X$, by Lemma 2.2 there exists a $\widetilde{T} \in \mathcal{K}(X, Z)$ with $\|\widetilde{T}\| \leq \mu\|T\|$, which is an extension of $T$. Since $X$ has the $\lambda$-BWAP, there exists an $S_{0} \in \mathcal{F}(X)$ with $\left\|S_{0}\right\| \leq \lambda\|i \widetilde{T}\|$, where $i: Z \rightarrow X$ is the inclusion map, such that

$$
\sup _{z \in K}\left\|S_{0} z-i T z\right\| \leq \frac{\varepsilon}{2 \mu+4} \text {. }
$$

Put $Y:=Z+S_{0}(X)$. Then $\operatorname{dim} Y / Z<\infty$. By Lemma 2.1 there exists a projection $P$ from $Y$ onto $Z$ with $\|P\| \leq 2 \mu+4$. Let $S:=\left.P S_{0}\right|_{Z}$. Then $S \in \mathcal{F}(Z)$ and for every $z \in K$

$$
\|S z-T z\|=\left\|P S_{0} z-P T z\right\| \leq \varepsilon
$$

and we also have

$$
\|S\| \leq(2 \mu+4) \lambda\|\widetilde{T}\| \leq(2 \mu+4) \mu \lambda\|T\|,
$$

hence $Z$ has the $(2 \mu+4) \mu \lambda$-BWAP.

\section{Proofs of Theorems 1.2 And 1.3}

We denote by $\|\cdot\|_{\pi}$ and $\|\cdot\|_{\mathcal{I}}$, respectively, the projective tensor norm and the integral ideal norm. The following lemmas are needed to prove Theorem 1.2.

Lemma 3.1. [6, Theorem 2.5] Let $T \in \mathcal{L}(X)$ and let $\lambda \geq 1$. The following statements are equivalent.

(a) $T \in \overline{\{S \in \mathcal{F}(X):\|S\| \leq \lambda\|T\|\}}{ }^{\tau_{c}}$.

(b) $\|T S\|_{\pi} \leq \lambda\|T\|\|S\|_{\mathcal{I}}$ for every $S \in \mathcal{F}(X)$.

(c) $\|T S\|_{\pi} \leq \lambda\|T\|\|S\|_{\mathcal{I}}$ for every Banach space $Y$ and every $S \in \mathcal{F}(Y, X)$.

Lemma 3.2. Let $\mathcal{A}(X)$ be a subset of $\mathcal{L}(X)$. Let $T \in \mathcal{L}(X)$ and let $\lambda \geq 1$. Then $T \in \overline{\{S \in \mathcal{A}(X):\|S\| \leq \lambda\|T\|\}}{ }^{\tau_{c}}$ if and only if for every finite-dimensional subspace $F$ of $X$ and every $\varepsilon>0$, there exists an $S \in \mathcal{A}(X)$ with $\|S\| \leq \lambda\|T\|$ such that $\|S x-T x\| \leq \varepsilon\|x\|$ for every $x \in F$.

Proof of Theorem 1.2. (a) $\Rightarrow$ (b) Let $Z$ be an ideal in $X$. Let $T \in \mathcal{K}(Z)$. We use Lemma 3.1(b) to prove that $T \in \overline{\{S \in \mathcal{F}(Z):\|S\| \leq \lambda\|T\|\}}{ }^{\tau_{c}}$. Let $j: Z \rightarrow X$ be the inclusion map and let $\Phi: Z^{*} \rightarrow X^{*}$ be a Hahn-Banach extension operator. For a Banach space $B$, we denote by $i_{B}$ the canonical isometry from $B$ to $B^{* *}$.

Now, since the operator $T^{*}: Z^{*} \rightarrow Z^{*}$ is weak ${ }^{*}$ to weak continuous, $T^{* *}$ maps from $Z^{* *}$ into $i_{Z}(Z)$. Thus the operator $i_{Z}^{-1} T^{* *} \Phi^{*} i_{X}: X \rightarrow Z$ is well defined and we claim that

$$
i_{Z}^{-1} T^{* *} \Phi^{*} i_{X} j=T \text {. }
$$

Indeed, for every $z \in Z$ and $z^{*} \in Z^{*}$,

$$
\Phi^{*} i_{X} j(z)\left(z^{*}\right)=i_{X} j(z)\left(\Phi\left(z^{*}\right)\right)=z^{*}(z)=i_{Z}(z)\left(z^{*}\right) .
$$

Then for every $z \in Z$, we have

$$
i_{Z}^{-1} T^{* *} \Phi^{*} i_{X} j(z)=i_{Z}^{-1} T^{* *} i_{Z}(z)=T z .
$$


Now, let $S \in \mathcal{F}(Z)$. Since $Z$ is an ideal in $X, Z^{*} \hat{\otimes}_{\pi} Z$ is a closed subspace of $Z^{*} \hat{\otimes}_{\pi} X$ (cf. [10, Theorem 3.4]). Thus $\|T S\|_{\pi}=\left\|j i_{Z}^{-1} T^{* *} \Phi^{*} i_{X} j S\right\|_{\pi}$. Since $X$ has the $\lambda$-BWAP, by Lemma 3.1(c) we have

$$
\begin{aligned}
\|T S\|_{\pi} & =\left\|j i_{Z}^{-1} T^{* *} \Phi^{*} i_{X} j S\right\|_{\pi} \\
& \leq \lambda\left\|j i_{Z}^{-1} T^{* *} \Phi^{*} i_{X}\right\|\|j S\|_{\mathcal{I}(Z, X)} \\
& \leq \lambda\|T\|\|S\|_{\mathcal{I}(Z, Z) .}
\end{aligned}
$$

$(\mathrm{b}) \Rightarrow$ (c) Let $Y$ be a separable closed subspace of $X$. Then by [11, Theorem] there exists a separable ideal $Z$ in $X$ such that $Y \subset Z$. Hence by (b) $Z$ has the $\lambda$-BWAP.

(c) $\Rightarrow$ (a) This proof is due to the one of $[4$, Lemma $3(\mathrm{~b})]$. Let $T \in \mathcal{K}(X)$. We use Lemma 3.2 to prove that $T \in \overline{\{S \in \mathcal{F}(X):\|S\| \leq \lambda\|T\|\}}{ }^{\tau_{c}}$. Let $F$ be a finite-dimensional subspace of $X$ and let $\varepsilon>0$ be given. Then by [9, Lemma 1] we see that there exists a separable subspace $Y$ of $X$ such that for every finitedimensional subspace $E$ of $X$ with $F \subset E$ there exists an operator $T_{E}: E \rightarrow Y$ satisfying that $\left\|T_{E}\right\| \leq 1+1 / \operatorname{dim} E$ and the restriction $\left.T_{E}\right|_{F}$ is the identity map. Consider the separable subspace $\overline{\operatorname{span}}(T(X) \cup Y)$ of $X$. Then by (c) there exists a separable closed subspace $Z$ of $X$ with $\overline{\operatorname{span}}(T(X) \cup Y) \subset Z$ such that $Z$ has the $\lambda$-BWAP. Since the restriction $\left.T\right|_{Z} \in \mathcal{K}(Z)$, there exists an $S \in \mathcal{F}(Z)$ with $\|S\| \leq \lambda\|T\|$ such that

$$
\|S f-T f\| \leq \varepsilon\|f\|
$$

for every $f \in F$. We define the map $S_{E}: X \rightarrow X$ by

$$
S_{E} x=S T_{E} x \text { if } x \in E, S_{E}=0 \text { otherwise, }
$$

for every finite-dimensional subspace $E$ of $X$ with $F \subset E$. By compactness, there is a subnet which converges pointwise to a finite rank linear operator $\widetilde{S}$ on $X$ with $\|\widetilde{S}\| \leq \lambda\|T\|$ and for every $f \in F$, we have

$$
\|\widetilde{S} f-T f\|=\lim _{G}\left\|S_{G} f-T f\right\|=\lim _{G}\left\|S T_{G} f-T f\right\|=\|S f-T f\| \leq \varepsilon\|f\| .
$$

Remark 3.3. In view of the proof of Theorem $1.2(\mathrm{a}) \Rightarrow(\mathrm{b})$, we see that for every $T \in \mathcal{W}(X)$, the space of all weakly compact operators on $X$,

$$
T \in \overline{\{S \in \mathcal{F}(X):\|S\| \leq \lambda\|T\|\}}^{\tau_{c}}
$$

if and only if for every ideal $Z$ in $X$, for every $T \in \mathcal{W}(Z)$,

$$
T \in \overline{\{S \in \mathcal{F}(Z):\|S\| \leq \lambda\|T\|\}}{ }^{\tau_{c}} .
$$

Proof of Theorem 1.3. (a) $\Rightarrow$ (b) follows from the proof of Theorem 1.1. (b) $\Rightarrow$ (c) is trivial. The proof of $(\mathrm{c}) \Rightarrow(\mathrm{d})$ is similar to $(\mathrm{b}) \Rightarrow(\mathrm{c})$ in Theorem 1.2.

$(d) \Rightarrow(a)$ This result was inspired from [4, Lemma 3(a)]. Let $T \in \mathcal{K}(X)$. Let $K$ be a compact subset of $X$ and let $\varepsilon>0$ be given. Consider the separable subspace $\overline{\operatorname{span}}(K \cup T(X))$ of $X$. Then by (c) there exists a separable closed subspace $Z$ 
of $X$ with $\overline{\operatorname{span}}(K \cup T(X)) \subset Z$ such that $Z$ has the WAP. Since the restriction $\left.T\right|_{Z} \in \mathcal{K}(Z)$, there exists an $S \in \mathcal{F}(Z)$ such that

$$
\sup _{x \in K}\|S x-T x\| \leq \varepsilon .
$$

Then by an application of the Hahn-Banach theorem there exists an extension $\widehat{S}: X \rightarrow Z$ of $S$. Then $j \widehat{S} \in \mathcal{F}(X)$, where $j: Z \rightarrow X$ is the inclusion map, and

$$
\sup _{x \in K}\|j \widehat{S} x-T x\|=\sup _{x \in K}\|S x-T x\| \leq \varepsilon .
$$

Hence $X$ has the WAP.

Acknowledgement. The authors would like to thank the referee for valuable comments. The first author was supported by NRF-2013R1A1A2A10058087 funded by the Korean Government.

\section{REFERENCES}

1. C. Choi and J.M. Kim, Weak and quasi approximation properties in Banach spaces, J. Math. Anal. Appl. 316 (2006), 722-735.

2. H. Fakhoury, Sélections linéaires associées au théorème de Hanh-Banach, J. Funct. Anal. 11 (1972), 436-452.

3. G. Godefroy and P.D. Saphar, Three-space problems for the approximation properties, Proc. Amer. Math. Soc. 105 (1989), 70-75.

4. W.B. Johnson, A complementary universal conjugate Banach space and its relation to the approximation problem, Israel J. Math. 13 (1972), 301-310.

5. N.J. Kalton, Locally complemented subspaces and $\mathcal{L}_{p}$-spaces for $0<p<1$, Math. Nachr. 115 (1984), 71-97.

6. J.M. Kim, A bounded approximation of weakly compact operators, J. Math. Anal. Appl. 401 (2013), 154-159.

7. J.M. Kim, On relations between weak approximation properties and their inheritances to subspaces, J. Math. Anal. Appl. 324 (2006), 721-727.

8. Å. Lima, V. Lima and E. Oja, Bounded approximation properties via integral and nuclear operators, Proc. Amer. Math. Soc. 138 (2010), 287-297.

9. J. Lindenstrauss, On nonseparable reflexive Banach spaces, Bull. Amer. Math. Soc. 72 (1966), 967-970.

10. E. Oja, Operators that are nuclear whenever they are nuclear for a larger range space, Proc. Edinburgh Math. Soc. 47 (2004), 679-694.

11. B. Sims and D. Yost, Linear Hanh-Banach extension operators, Proc. Edinburgh Math. Soc. 32 (1989), 53-57.

${ }^{1}$ Department of Mathematical Sciences, Seoul National University, Seoul, 151-747, KOREA.

E-mail address: kjm21@kaist.ac.kr

${ }^{2}$ Institute for Ubiquitous Information Technology and Applications, Konkuk University, Seoul, 143-701, Korea.

E-mail address: northstar@kaist.ac.kr 